\title{
Effective Car Parking Reservation System Based on Internet of things Technologies
}

\author{
PavanKumar Jogada and Vinayak Warad
}

\begin{abstract}
Nowadays with the rapid increase in urban population, there is a major problem with the parking System in almost every major city across the globe. Many of us get highly disturbed when there is no proper space for parking space for our vehicles. In this article, we have proposed a smart parking application, where users will be able to park their automobiles by finding an empty parking slot through Android Application or can even park their automobiles directly through Embedded Hardware. An Intelligent Parking System is implemented based on Slot Allotment. There are two modes using which the Android user can book the parking slots easily like Advance \& Current Booking. Using these modes the application user can also choose the easiest and nearest route and destination. The Android Application itself will serve as a payment gateway. Embedded Hardware is implemented for Direct Parking. Server will monitor the Slot Allotment dynamically.
\end{abstract}

Index Terms--- IoT (Internet of Things), Infrared sensor, RFID (Radio-Frequency Identification), Wireless sensor, Wireless sensor network (WSN), ZigBee.

\section{INTRODUCTION}

$\mathrm{T}$ HE emanation in Information and Communication Technology (ICT) such as wireless sensors has introduced new and very existing applications in every part of life. These applications either from machine-to-machine (M2M) or person-to-machine (P2M) have enhanced the living standards. Besides it has included the advantages of reduced better security and more sustainable technology. Wireless sensor network (WSN) is very evident in the current research and development literature in terms of smart cities where the various applications are integrated together with the help of the improved technology. Nowadays with the increased population in the cities, car parking has become a major issue. This issue can be overcome by the modern technology. In the context, an android application is developed for smart car parking. In this android application, slots can be booked for parking the vehicle. Booking can be done in three ways as Current booking, advanced booking and direct booking. Online payment can be done for the booked slots using the android application. There is a RFID (Radio-frequency Identifier) that is used for confirming that the booked vehicle has arrived in the particular slot. Radiofrequency identification (RFID) uses the electromagnetic fields to transfer data

PavanKumar Jogada, Dept. of CSE, SKSVMACET, Laxmeshwar, India. E-mail:pavankumarj34@gmail.com

Vinayak Warad, Dept. of CSE, SKSVMACET, Laxmeshwar, India. E-mail:vinaywarad1995@Gmail.Com

DOI:10.9756/BIJSESC.8261 automatically by identifying and tracking the attached objects. These tags will contain the electronically stored information. Some types of tags collect energy by interrogating radio waves and they serve as a passive transponder. The user will also specify the time period for which he needs the slot to be booked.[1]

\section{SYSTEM ARCHITECTURE}

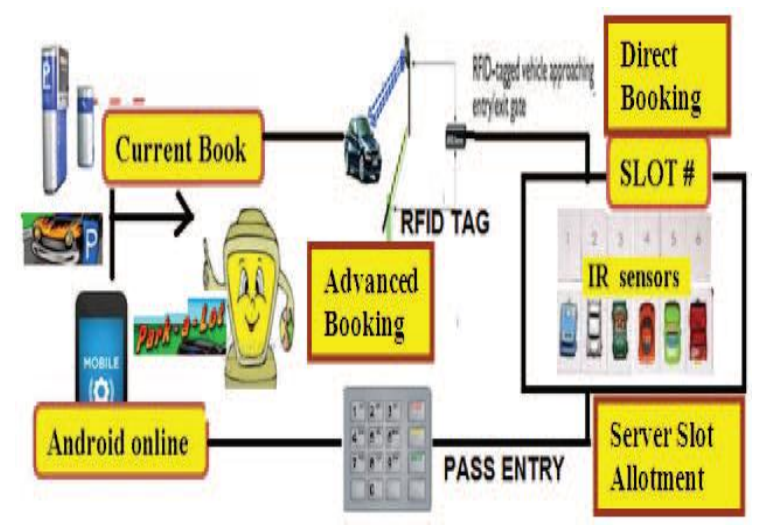

The effective parking reservation system is a very efficient technology in overcrowded places. Our proposed system is a smart way of reserving the parking slot using Android application. The IR sensors are used to detect the availability of the slots. The proposed system contains two phases. First is Android implementation and second is Embedded Hardware implementation.[2][3][4]

\section{A. Phase 1: Android Implementation}

In android implementation there are two modules namely Current Booking and Advanced Booking, We create an Android application and deploy it in user's phone. The server will list out the entire available parking slot in the particular place. Mobile user will select the parking slot and also fix approximate time. This is also updated in the server of the parking space and grace time will be provided. In Current Booking module, the mobile user will be able to book their parking slot only for a particular time in that particular day whereas in Advanced Booking module the mobile user will be able to book the parking slot for their vehicle well in advance of time. The user should specify their vehicle number and the time period for which they need the slot to be booked while booking. The android application itself serves as a payment gateway for both the Current and Advanced booking modules. After the payment is made for the parking slot the status of that particular slot is updated in the server and makes it not available for the other people during the booked timing. A certain amount of grace time is given for the slot booker. If the particular vehicle does not reach the parking slot booked 
within the grace time limit, the slot will be made available to the others and the payment gets reverted.

\section{B. Phase 2: Embedded Hardware Implementation}

In the second phase the user will go directly to the parking space slot and show their RFID TAG. The vehicle consists of a RFID tag. This is read by the reader in the gate section. This value is transmitted to the parking slot section through a zigbee transceiver or Li-Fi transceiver. A microcontroller in the gate section stores and updates the empty slot's number whenever a vehicle leaves a parking slot. A RFID tag corresponding to this free parking slot is provided to the vehicle which is entered in the gate section earlier. The vehicle then goes to the parking slot Section. This section has a RFID reader to verify both the vehicle's tag and the empty slot's tag. So the user will be able to park their vehicle in the free slots. This will be updated in the server. IR sensor is used here to detect whether the slot is free or not. The sensor which is interfaced with controller is also updated.[5][6]

\section{SOME IMPORTANT COMPONENTS}

Some of the important components which are used in the proposed system are discussed briefly in this section.

A. Parking Lot Section

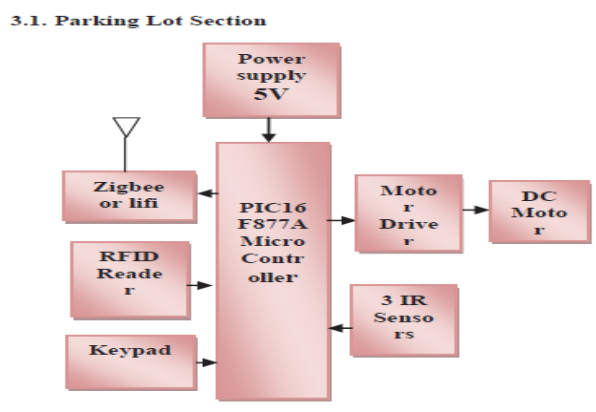

\section{B. Zigbee}

Bluetooth has a very limited range and has a relatively low bandwidth. These Bluetooth devices are mainly used for the handheld systems. Due to their limited range and speed they are mostly not used in wireless local-area network (WLAN) networking applications. Wi-Fi supports Ad-Hoc mode and it is used as a public hotspot. 802.11a does not support cross compatibility. The speed is different for varying distances. The main aim of this paper is to find a parking slot using the smart car parking system. ZigBee technology has the advantages of low power consumption, low data rate, and its of low cost. ZigBee is implemented in larger mesh networks. The ZigBee has different data rates of $250 \mathrm{kbps}$ at $2.4 \mathrm{GHz}$; $40 \mathrm{kbps}$ at $915 \mathrm{MHz}$ and $20 \mathrm{kbps}$ at $868 \mathrm{MHz}$. IEEE and ZigBee Alliance have been ranking to specify the entire protocol stack. IEEE 802.15.4 aims to focus on specification of the lower two layers of the protocol (physical and data link layer). ZigBee focuses to provide interoperable data networking for the upper protocol stack. It also aims at providing security services and wireless home and building control systems, compliance testing and evolution of standard for advanced engineering. This will guarantee the customers to buy products from various manufacturers with a hope that these products will work efficiently.[7]

\section{Infrared Sensors}

The aim of infrared sensors is to find availability of the slot for parking the vehicle. These Infrared Sensors are detected using remote control. These are generally used to listen to the infrared light. Due to radiation these objects emit heat energy and can be detected by using electronic devices, which are not visible to human eyes. The infrared sensors generate energy when exposed to heat. The sensor will detect change in the infrared radiation .If the value of the infrared radiation is higher than a predefined value then free slot information Is given to the microcontroller. A pulse of the infrared light is generated by the IR sensor and is emitted from the emitter. IR light is reflected when the object is detected. Some of the infrared light will hit the detector and thus it is known whether there is any space available for car parking. [8]

\section{Internet of Things}

Internet of Things (IoT) is used for interconnecting the embedded computing devices that are individually identifiable in the existing internet infrastructure. IoT will typically offer advanced connectivity with various devices. It will provide beyond Machine-To-Machine communications (M2M) thereby covering various protocol, domains and real-time applications. Automation in all fields is possible with the help of the interconnections of the embedded devices which enables advanced applications like Smart Grid. [9][10]

\section{E. PIC16F877A Microcontroller}

This microcontroller is an easy-to-program (only 35 single word instructions) CMOS FLASH-based 8-bit microcontroller. It is highly compatible with the PIC16C5X, PIC12CXXX and PIC16C7X devices. It features the 256 bytes of EEPROM data memory. This microcontroller is self programmable. It has an ICD, 2 Comparators, 8 channels of A/D Converters, an Inter-Integrated Circuit bus, a parallel slave port and a Universal Asynchronous Receiver Transmitter (USART). These advanced features make it ideal for use in the consumer, industrial and automotive information. This microcontroller is used in embedded part of the parking system.[11][12]

\section{F. Radio-Frequency Identification (RFID)}

Radio-frequency identification (RFID) makes use of the electromagnetic fields for the transformation of the data that automatically identifies and tracks the tags attached to various objects. These tags will contain electronically stored information. Energy is collected from the radio waves which are interrogated by the tags and acts as a passive Transponder. Automatic Identification and Data Capture (AIDC) is possible with the help of RFID.RFID reader is used for transmitting radio signal that is encoded to interrogate the tag. The RFID tag responds with identification after receiving the vehicle consists of a RFID tag. This is read by the reader in the Gate section. This value is transmitted to the parking slot section through a zigbee transceiver or $\mathrm{Li}-\mathrm{Fi}$ transceiver. A microcontroller in the gate section stores and updates the empty slot's number whenever a vehicle leaves a parking slot. A RFID tag corresponding to this free parking slot is provided 
to the vehicle which entered the gate section earlier. The vehicle then goes to the parking slot section. This section has a RFID reader to verify the vehicle's tag and the empty slot's tag. So the user can be parked their vehicle in the free slots. This will be updated in the server.[13]

\section{G. Server Section}

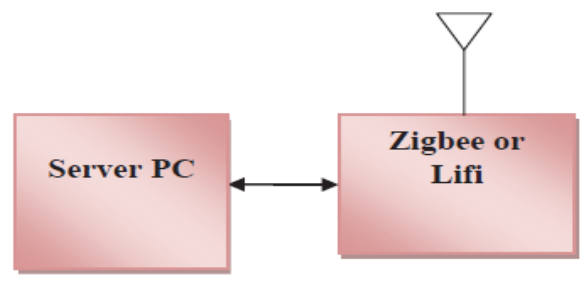

The Server PC is used to store the availability status of all the parking slots in that area. The slots get allocated dynamically. Each and every time there is a change in the status of the parking slot there is an updating process done on the server pc to provide accurate details of the availability of the slots.[14]

\section{CONCLUSION AND Future SCOPE}

There is a great difficulty met by the people today in finding an empty slot for parking their vehicles. A lot of time is spent to find an empty slot for parking the car. In this context, we develop an effective parking reservation system where the user can book their particular slot using their android application or with the help of an embedded hardware. This system is highly reliable and efficient. This system can be used to avoid heavy traffic in the parking areas like shopping malls, theatres, tourist spots and other busy areas thereby reducing time and the consumption of the fuel and pollution. Cyber security to the parking cloud service has to be implemented in our future endeavors as there are many security risks involved in real time implementations of both cloud server and IoT.[15]

\section{REFERENCES}

[1] F. Losilla, A.J Garcia-Sanchez, F. Garcia-Sanchez and J. Garcia- Haro, "Onthe Role of Wireless Sensor Networks in intelligent transportation Systems, ICTON, Pp. 2161-2056, 2012.

[2] J. Chinrungrueng, S. Dumnin and R. Pongthornseri, "I Parking: A Parking Management Framework", 11th International conference on ITS Telecommunications, Pp. 63-68, 2011.

[3] Y. Hirakata, A. Nakamura, K. Ohno and M. Itami, "Navigations Systemusing Zigbee Wireless Sensor Network for Parking", 12 International Conference on ITS Telecommunications, Pp. 605-609, 2012

[4] W. Lumpkins, "The internet of things meets cloud computing," IEEE Consum. Electron. Mag., Vol. 2, No. 2, Pp. 47-51, 2013.

[5] A. Aijaz and A.H. Aghvami, "Cognitive Machine-to-Machine Communications for Internet-of-Things: A Protocol Stack Perspective", Internet of Things Journal, IEEE, 2015.

[6] D. Silva, P. Bartolomeu and J. Fonseca, "Wireless Parking Lot Monitoring and Guidance", 16th IEEE Conference on Engineering tech. \& Factory Automation, Pp. 1-49, 2011.

[7] M. A. Feki, F. Kawsar, M. Boussard and L. Trappeniers, "The Internet of Things: The next technological revolution," Computer, Vol. 46, No. 2.

[8] N.H.H.M. Hanif, M.H. Badiozaman and H. Daud, "Smart parking reservation system using short message services (SMS)", Intelligent and Advanced Systems (ICIAS), Pp. 1-5, 2010.
[9] L. Atzori, A. Iera and G. Morabito, "The internet of things: A survey", Computer networks, Vol. 54, No. 15, Pp. 2787-2805, 2010.

[10] M. Palattella, "Standardized protocol stack for the Internet of (important) Things", IEEE Communications Surveys \& Tutorials, Vol. 15 , No. 3 , Pp.1389 -1406, 2013.

[11] J. Rudell, V. Bhagavatula and W. Wesson, "Future integrated sensor radios for long-haul communication", Vol. 52 , No. 4 , Pp. 101 -109, 2014

[12] A. Zanella, N. Bui, A. Castellani and L. Vangelista, "Internet of Things for Smart Cities" IEEE, Vol. 1, No. 1, 2014.

[13] Y. Dong, A. Wickramasinghe, H. Xue, S.F. Al -Sarawi and D.C. Ranasinghe, "A novel hybrid powered RFID sensor tag", In 2015 IEEE International Conference on RFID (RFID), Pp. 55-62, 2015.

[14] G. Lasser and C.F. Mecklenbrauker, "Self-interference noise limitations of RFID readers", IEEE Conference on RFID, Pp. 145-150, 2015.

[15] M.A. Razzaque, M. Milojevic-Jevric, A. Palade and S. Clarke, "Middleware for internet of things: a survey", IEEE Internet of Things Journal, Vol. 3, No. 1, Pp. 70-95, 2016.

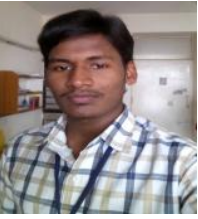

PavanKumar Jogada

Computer science and engineering

SKSVMACET-Laxmeshwar

pavankumarj34@gmail.com

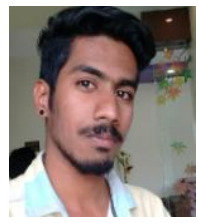

Vinayak.Warad, Computer science and engineering, SKSVMACET-Laxmeshwar

vinaywarad1995@gmail.com 